\title{
NADH Dehydrogenase (Ubiquinone)
}

National Cancer Institute

\section{Source}

National Cancer Institute. NADH Dehydrogenase (Ubiquinone). NCI Thesaurus. Code C118184.

A complex of over 40 proteins found in the inner mitochondrial membrane. This protein complex catalyzes the transfer of electrons from NADH to ubiquinone (coenzyme Q10) and plays a role in the initiation of the mitochondrial electron transport chain. 